\title{
Prueba de caminata de seis minutos
}

\author{
MÓNICA GUTIÉRREZ-CLAVERÍA* (Coordinadora), TERESA BEROÍZA W.*, \\ CLAUDIA CARTAGENA S.*, IVÁN CAVIEDES S.*, JUAN CÉSPEDES G.*, MÓNICA GUTIÉRREZ-NAVAS*, \\ MANUEL OYARZÚN G.*, SYLVIA PALACIOS M.* y PATRICIA SCHÖNFFELDT G.*
}

\section{Guidelines for the six-minute walk test}

The six-minute walk test has been shown as a very useful tool in the functional assessment of patients with chronic respiratory diseases enclosing patients with pulmonary hypertension. Methodological standardization of this test is fundamental for interpreting its results, as well as for using it in the short and long-term clinical follow up of our patients. The purpose of these guidelines is justly to spread out in our country the proper way to perform this useful test. In this context, indications, contraindications, limitations, security measures and detailed instructions about how to carry out, how to report and how to interpret the 6 minute walk test are described in these guidelines.

Key words: 6 min walk test, standardized methodology, interpretation.

\section{Resumen}

La prueba de caminata de 6 minutos ha demostrado ser una herramienta muy útil en la evaluación funcional de los pacientes con enfermedades respiratorias crónicas, incluyendo pacientes con hipertensión pulmonar. Para su correcta interpretación y uso clínico en el seguimiento de pacientes, es fundamental estandarizar la técnica. El propósito de este instructivo es justamente difundir a nivel nacional, la manera de efectuar esta técnica en forma correcta. En este contexto, este instructivo describe las indicaciones, contraindicaciones, limitaciones, medidas de seguridad y entrega detalles sobre la ejecución, informe e interpretación de la prueba de caminata de 6 minutos.

Palabras clave: Prueba caminata 6 min, estandarización, interpretación.

\section{Introducción}

Tradicionalmente para evaluar la función respiratoria se hacen pruebas en condiciones de reposo, desde las primeras mediciones efectuadas a mediados del s. XIX de lo que hoy llamamos capacidad vital hasta la actual curva de relación flujo/volumen o el estudio de volúmenes por pletismografía. Sin embargo, la actividad humana se realiza fundamentalmente en movimiento, haciendo esfuerzos que ponen en condición de estrés tanto al sistema respiratorio como al cardiovascular y al músculo-esquelético. En la década de los "70 se dio a conocer el test de Cooper ${ }^{1}$ o prueba de carrera de 12 minutos, de gran aplicabilidad en la evaluación de la condición física en deportistas, pero muy exi- gente en sujetos con patologías cardíacas o respiratorias, por lo cual aparecieron modificaciones como las sugeridas por Mc Gavin y cols, en $1976^{2}$, que la transforma en caminata y especialmente por la prueba reducida a 6 minutos de caminata (PC6min) presentada en 1982 por Butland y cols ${ }^{3}$, en pacientes respiratorios. Allí se demuestra su utilidad como método de evaluación en un sistema más adecuado al paciente, más natural y más fácil de controlar por el equipo de salud.

Desde entonces aparecen numerosas publicaciones que demuestran la utilidad de esta prueba tanto en pacientes respiratorios como cardíacos, estando especialmente indicada en aquellos de mayor compromiso, con valor pronóstico en mortalidad y morbilidad ${ }^{4}$ y que puede ser más

* Sección Función Pulmonar Adultos, Sociedad Chilena de Enfermedades Respiratorias. 11 de Diciembre, 2008. 
sensible para objetivar la desaturación en pacientes con enfermedad pulmonar obstructiva crónica $(\text { EPOC })^{5}$. Es especialmente de utilidad en el seguimiento de medidas terapéuticas y de rehabilitación e incluso en el trasplante pulmonar $^{6-10}$ ya que refleja más adecuadamente las limitaciones en las actividades de la vida diaria de las personas.

La estandarización actual y las fórmulas de regresión para conocer los límites normales de la PC6min hacen de ésta un instrumento de evaluación de gran rendimiento y con una relación costo-beneficio muy alta, transformándola en una herramienta de gran utilización en clínica, por lo cual nuestra Sociedad a través de su Sección de Función Pulmonar en Adultos, ha decidido dar los pasos necesarios para su estandarización en nuestro país. Este instructivo da a conocer las recomendaciones que deben tenerse presente al efectuar la PC6min.

\section{Objetivos}

Realizar la PC6min tiene como principal meta efectuar una evaluación objetiva de la capacidad funcional para hacer ejercicio, que vaya más allá de la tradicional pregunta acerca de cuántas cuadras camina o cuántos pisos sube en una escalera, que tiene respuestas muy subjetivas.

Se debe realizar en pacientes con moderada o severa limitación al ejercicio, ya sea de causa respiratoria o cardíaca y no es sustituto sino complemento del test cardio-pulmonar, ya que no puede diferenciar las causas que provocan la disnea como lo hace este último?.

A la utilidad clínica ya conocida se agrega su uso cada vez mayor en protocolos de investiga- ción, que irán aportando nuevas y mejores utilidades para esta prueba.

\section{Indicaciones}

La indicación más clara se relaciona con la medición de respuesta a intervenciones médicas en pacientes con enfermedad cardíaca o pulmonar de grado moderado a avanzado. También ha sido usada como una medición única del estado funcional del paciente, así como predictor de muerte y de morbilidad (Tabla 1).

\section{Limitaciones}

Los pacientes con alguna limitación funcional crónica para deambular podrían realizar el examen con los elementos de ayuda que habitualmente usen (bastones, prótesis, etc). En este caso no se deberá relacionar con los valores teóricos de población sana.

\section{Contraindicaciones}

Contraindicaciones absolutas

- Angina inestable en el primer mes de evolución.

- Infarto agudo del miocardio en el primer mes de evolución.

- Imposibilidad para caminar por evento agudo (v. gr. esguince de tobillo, herida en el pie, fractura de pierna, etc).

\section{Contraindicaciones relativas}

- Frecuencia cardíaca > 120 por minuto en reposo.

- Presión arterial sistólica $>180 \mathrm{mmHg}$.

- Presión arterial diastólica $>100 \mathrm{mmHg}$.

- Saturación arterial de oxígeno en reposo < 89\%.

Tabla 1. Indicaciones para la prueba de caminata de 6 minutos en la práctica clínica y su grado de recomendación ${ }^{11}$

\section{Indicación}

Diagnóstico de desaturación arterial con el ejercicio

Evaluación funcional de pacientes con EPOC, EPD, HPP e ICC

Evaluación de pronóstico de pacientes con EPOC, EPD, HPP e ICC

Evaluación funcional de pacientes con fibrosis quística

Evaluación de pronóstico de pacientes con EPOC o ICC previo a la cirugía (cirugía de reducción de volumen pulmonar, trasplante)

Evaluación de los beneficios de intervenciones terapéuticas (oxígeno suplementario, rehabilitación, cirugía)

EPOC: Enfermedad pulmonar obstructiva crónica; EPD: Enfermedad pulmonar intersticial difusa; HPP: Hipertensión Pulmonar Primaria; ICC: Insuficiencia cardíaca congestiva.

* Nivel de recomendación según las normas de la Medicina basada en evidencias.
Recomendación*

B


Medidas de seguridad en la prueba de caminata de seis minutos

Este examen debe realizarse en hospitales tipo I o II, bajo la tuición del especialista en enfermedades respiratorias. El lugar donde se realiza la prueba debe disponer de medidas de seguridad para el manejo de eventuales urgencias. Debe existir un timbre de ayuda ante situaciones de emergencia y hay que contar con un carro de reanimación cardiorrespiratoria de fácil y rápido acceso.

Disponer de los siguientes suministros: oxígeno, salbutamol inhalador, nitroglicerina sublingual, aspirina.

El técnico que realiza el examen debe ser un profesional adiestrado en reconocer emergencias críticas.

El médico no requiere estar presente durante todo el procedimiento. Excepción a esto lo constituyen la petición del médico tratante o si el médico, a cargo de la técnica, lo considera necesario ante un paciente determinado.

Si el paciente está recibiendo oxigenoterapia debe continuar con la dosis indicada.

Si el paciente utiliza elementos de ayuda para caminar, los debe usar durante la prueba.

Si las normas del hospital lo requieren, el paciente debe firmar un consentimiento informado antes de efectuar el procedimiento.

\section{Causas de detención o suspensión de la prueba}

- Dolor torácico.

- Disnea intolerable.

- Calambres intensos en las piernas.

- Diaforesis.

- Aparición de cianosis evidente.

- Palidez y aspecto extenuado.

Si el examen necesita ser suspendido por los motivos antes citados, el paciente debe ser sentado o acostado dependiendo de la severidad del evento y del riesgo de síncope. Además se debe controlar pulso, presión arterial, frecuencia respiratoria, saturación de oxígeno y avisar al médico encargado, si la situación así lo requiere.

Lugar físico, equipamiento y preparación del paciente

Lugar físico

- Pasillo interior recto y plano, de superficie dura, en lo posible de poco tránsito y con los elementos de privacidad adecuados.

- El pasillo debe tener idealmente 30 metros de longitud (mínimo aceptable: 20 metros).
- Marcar el pasillo cada 3 metros.

- Los puntos extremos del corredor deben ser señalizados con conos de colores.

- Marcar el inicio con una cinta adhesiva brillante y colorida.

- El ambiente debe tener temperatura y humedad agradables.

\section{Equipamiento requerido}

1. Cronómetro.

2. Conos de color para marcar puntos extremos del pasillo.

3. Sillas ubicadas de forma que el paciente pueda descansar.

4. Planilla de registro.

5. Oxímetro de pulso.

6. Esfigmomanómetro y estetoscopio.

7. Escala de Borg ${ }^{11}$ modificada plastificada.

8. Cinta adhesiva o adhesivos de color para marcar lugar de detención del paciente a los 6 min.

9. Tubo portátil de oxígeno.

10. Teléfono cerca.

11. Equipo de reanimación y camilla cerca.

12. Silla de ruedas disponible.

\section{Preparación del paciente}

Estas instrucciones deben ser entregadas por escrito previamente (anexo 1):

1. Vestir ropa cómoda holgada.

2. Usar zapatos planos apropiados para caminata rápida.

3. No suspender los medicamentos que usa habitualmente.

4. Comer liviano antes del examen: ingerir un desayuno liviano si el estudio es en la mañana o un almuerzo liviano si el estudio es en la tarde.

5. No hacer ejercicio 2 horas antes de la realización del examen.

\section{Instrucciones para la realización del examen}

El examen consiste en medir la distancia que puede caminar una persona en 6 minutos, habiéndole solicitado que recorra la mayor distancia posible en este tiempo. Se evaluará la presencia de disnea, frecuencia cardíaca, frecuencia respiratoria y saturación de oxígeno $\left(\mathrm{SpO}_{2}\right)$ al inicio e inmediatamente al final de la prueba.

Si el examen es uno de control, se deberá hacer aproximadamente a la misma hora que el anterior para minimizar la variabilidad dentro del día. 
- Deje al paciente en reposo sentado por 10 minutos. Si el pasillo está muy alejado, se puede incorporar el uso de una silla de ruedas para el traslado al lugar de realización del examen.

- En la hoja para registro anote los datos personales y después agregue las variables que usted mida: presión arterial, $\mathrm{SpO}_{2}$, la frecuencia respiratoria y el pulso.

- Realice cuestionario (anexo 2) al paciente para asegurarse que no tenga contraindicaciones.

- Explique brevemente qué hará el paciente y qué medirá usted durante el examen.

- Demuéstrele cómo dar la vuelta alrededor del cono.

- Muéstrele la Escala de Borg ${ }^{11}$ (anexo 3) y pídale que indique su nivel de fatiga y disnea en la escala. Asegúrese de que el paciente haya comprendido cómo indicará la magnitud de su disnea.

- Mida la $\mathrm{SpO}_{2}$.

- Inicie la caminata con el cronómetro programado para 6 minutos, sin detenerlo hasta terminar el examen.

- Anote cada vuelta en su hoja de registro.

- Estimule verbalmente al paciente cada $1 \mathrm{mi}-$ nuto según lo indicado (anexo 4), para que continúe caminando la máxima distancia que él pueda en 6 minutos.

- Termine el examen:

a) Al completar 6 minutos desde el inicio del examen.

b) Antes de completar los 6 minutos, si:

- El paciente no puede continuar.

- Usted estima que no debe continuar, de acuerdo a lo descrito en la guía.

- De inmediato mida la $\mathrm{SpO}_{2}$, la frecuencia respiratoria y la frecuencia cardíaca, anótelas, al mismo tiempo que el paciente indica en la escala de Borg ${ }^{11}$ cuál es la magnitud de su disnea y después cuál es la magnitud de su fatiga.

- Camine con el paciente hasta una silla para que descanse 10 minutos. Si en cualquier momento aparecen síntomas o signos de alarma, evalúe al paciente de inmediato, en reposo, y solicite la atención médica en caso de persistencia o mayor gravedad de sus síntomas o signos, según fue descrito en el protocolo de este examen.

- Mida la frecuencia respiratoria, el pulso, la presión arterial y la $\mathrm{SpO}_{2}$ a los 2 y 5 minutos de terminada la prueba, anótelos en su registro.

- Si después de descansar 10 min el paciente está en su condición basal, estable y sin sín- tomas ni signos de alarma, el examen está terminado.

\section{Instrucciones al paciente}

I. En primer lugar explique al paciente en qué consiste la prueba y qué utilidad tiene: "Esta prueba permite evaluar en forma global la respuesta de su cuerpo al ejercicio". "Refleja su nivel funcional para las actividades cotidianas".

II. Es muy importante que las instrucciones sean precisas y que el paciente se sienta cómodo y no atemorizado por el examen.

III. Posteriormente instruya al paciente así: "EI objetivo de este examen es que camine la mayor distancia posible durante 6 minutos. Usted caminará rápido de ida y vuelta en este pasillo. Seis minutos es un tiempo largo para caminar, de modo que se va a tener que esforzar. Probablemente usted se va a sentir muy cansado o con sensación de falta de aire. Puede ir más lento, detenerse y descansar sólo si es necesario. Se puede afirmar en la pared, pero deberá volver a caminar tan pronto como le sea posible. Durante el examen no debe conversar, para no alterar su concentración y rendimiento".

IV. Demuestre cómo se efectúa la marcha.

\section{Interpretación}

Para interpretar los resultados de este examen se considerará los valores absolutos de distancia caminada medidos y su expresión en relación a los valores normales publicados.

El valor aislado en la evaluación de un paciente con EPOC permite conocer su capacidad funcional durante un ejercicio submáximo, similar al que podría desarrollar en su vida diaria ${ }^{12}$.

En un estudio reciente en pacientes con EPOC que fueron seguidos por 55 meses $^{13}$, se ha descrito un valor umbral para mortalidad en ellos. Así, la mortalidad debida a EPOC en pacientes con enfermedad moderada o grave, fue de $39 \%$ si caminaron menos del $54 \%$ del valor de referencia, para distancia caminada en 6 minutos calculado por la ecuación de Troosters y de un $38 \%$ si caminaron menos del $67 \%$ del valor predicho por la ecuación de Enright. La mortalidad por EPOC en los pacientes que caminaron distancias mayores fue de $12 \%$ en el análisis con cualquiera de las dos ecuaciones.

La expresión de este valor absoluto también permite distinguir grupos de pacientes que tienen distinto pronóstico. Así, se ha descrito que los pacientes con EPOC que caminaron menos 
de 350 metros tuvieron una mortalidad, debida a EPOC, más elevada que aquellos que caminaron una mayor distancia (mortalidad 40 versus $11 \%$ ).

En pacientes con EPOC avanzada, sometidos a cirugía de reducción de volumen pulmonar ${ }^{14}$, se ha descrito que si en la evaluación pre-operatoria caminaron más de 200 metros y no tuvieron hipercapnia, su estadía hospitalaria era menor de 3 semanas y sobrevivieron a los 6 meses de seguimiento. Entre los pacientes que en la evaluación pre-operatoria tenían valores menores de distancia caminada en 6 min o tenían hipercapnia hubo una evolución considerada inaceptable, por tener una hospitalización prolongada o por fallecer en los primeros 6 meses después de la cirugía.

En la práctica clínica, además de obtener una medición basal aislada de distancia caminada en 6 minutos, el propósito de repetir este examen, es responder a la pregunta de cuánto ha mejorado un paciente después de una intervención.

Esta mejoría será clínicamente significativa cuando sea al menos igual al valor de mínima diferencia importante.

Dado que la investigación en este tema está en desarrollo, recientemente se ha publicado un estudio $^{15}$ que concluye que un cambio en al menos 35 metros, en la distancia caminada en 6 min, es un cambio relevante en pacientes con EPOC moderada y avanzada. Este valor, (distancia caminada en metros después de la intervención-distancia caminada en metros antes de la intervención), se expresará como valor absoluto en metros y será usado para interpretar la situación clínica por el médico tratante.

La disminución anual de los valores de distancia caminada, en pacientes con EPOC avanzada, que es más marcada en pacientes con peor función pulmonar, podría ser una herramienta de seguimiento de la evolución de estos pacientes, ya que continúa disminuyendo en los pacientes más graves, a pesar de que la función pulmonar se mantenga estable ${ }^{16}$.

La integración de los valores de este examen en el índice BODE ${ }^{17}$ (índice de masa corporal, $\%$ de $\mathrm{VEF}_{1}$, disnea, distancia caminada en 6 min), ha permitido categorizar y predecir pronóstico en pacientes con EPOC. Se ha demostrado que este índice es un mejor predictor de mortalidad que el $\mathrm{VEF}_{1}$ en pacientes con EPOC.

Este examen ha permitido indicar oxígenoterapia en los pacientes con EPOC que sólo tienen disnea e hipoxemia durante el ejercicio, así como también recomendar la rehabilitación o la mantención de actividad física en estos pacientes $^{18}$.

\section{Valor pronóstico en otras enfermedades respiratorias}

En un estudio sobre valor pronóstico de la distancia caminada en 6 min en pacientes con hipertensión pulmonar primaria ${ }^{19}$, se ha descrito una sobrevida de $20 \%$ a 20 meses para los pacientes que caminaron menos de $332 \mathrm{~m}$ y de $90 \%$ para los pacientes que caminaron distancias mayores.

En pacientes con enfermedad pulmonar difusa, en lista de espera para trasplante pulmonar ${ }^{20}$, se ha observado que aquellos que caminan menos de 207 m tienen una mortalidad que es más de 4 veces la mortalidad de los que caminan distancias mayores. En este estudio este valor fue un mejor predictor de mortalidad a los 6 meses que la capacidad vital forzada expresada como porcentaje del valor teórico.

\section{Distancia caminada, medición de otras variables \\ Saturación de Oxígeno $\left(\mathrm{SpO}_{2}\right)$}

Si bien la medición de la saturación de hemoglobina es considerada opcional en el Consenso de la American Thoracic Society (ATS), actualmente está incorporada en este examen para propósitos clínicos y de investigación.

La oximetría de pulso debe ser incluida porque es necesaria para los aspectos de seguridad del examen, para evitar exponer a los pacientes a hipoxemia. Una caída significativa de $\mathrm{SpO}_{2}$ es aquella mayor o igual a $4 \%{ }^{21}$.

Se ha destacado su utilidad en el diagnóstico de hipoxemia en ejercicio, en la demostración del aumento de la distancia caminada con administración de oxígeno y en la indicación y evaluación de rehabilitación y de oxigenoterapia en pacientes con $\mathrm{EPOC}^{18}$.

La disminución de $4 \%$ de la $\mathrm{SpO}_{2}$ en pacientes con EPOC con $\mathrm{PaO}_{2}$ basal de al menos 60 $\mathrm{mmHg}$ podría tener algún papel complementario a la distancia caminada como predictor de la mortalidad en pacientes con enfermedad avanzada. En 2008, Casanova y cols ${ }^{22}$, observaron una mortalidad de $67 \%$ en pacientes con desaturación y de $38 \%$ en pacientes sin desaturación a los 8 años de seguimiento.

Se ha demostrado que la disminución de $\mathrm{SpO}_{2}$, a $88 \%$ o menos al terminar el examen, es un muy buen predictor de mortalidad en pacientes con enfermedad pulmonar difusa fibrótica. Lama y $\operatorname{cols}^{23}$, en pacientes con fibrosis pulmonar idiopática, observados durante 4 años, demostraron que aquellos pacientes cuya $\mathrm{SpO}_{2}$ fue menor de $88 \%$ tuvieron una probabilidad de morir 
cuatro veces mayor que la de aquellos que no tuvieron esa caída en la $\mathrm{SpO}_{2}$. Esta variable, caída de la $\mathrm{SpO}_{2}$ a $88 \%$ o menos al terminar la caminata de $6 \mathrm{~min}$, es muy reproducible, al medir la variabilidad intrasujeto en pacientes con enfermedad pulmonar difusa fibrótica ${ }^{24}$.

\section{Frecuencia de pulso}

La inclusión de la frecuencia de pulso arterial permite controlar aspectos de la seguridad del examen y complementar la información acerca de los cambios fisiológicos que se asocian a la mejoría en la tolerancia al ejercicio.

\section{Disnea y fatiga en las piernas}

Esta prueba permite objetivar los síntomas disnea y fatiga en las piernas, medidos por la escala de Borg. Esta información es útil para la interpretación de los mecanismos fisiológicos que acompañan a los cambios del rendimiento en la prueba después de una intervención.

\section{Informe}

Este examen será informado por el médico especialista en Enfermedades Respiratorias.

Se sugiere usar una plantilla de informe (anexo 5) donde se registren los valores de frecuencia de pulso, frecuencia respiratoria, $\mathrm{SpO}_{2} \%$, disnea y fatiga en las piernas (escala de Borg), al inicio de la prueba y a los 6 min. Se anotarán los valores absolutos de distancia caminada (m) y se recomienda expresarlos además como \% de los valores de referencia. Se recomienda además medir y registrar el pulso, frecuencia respiratoria, presión arterial, $\mathrm{SpO}_{2}$ a los 2 y 5 minutos de terminada la prueba, mientras el paciente descansa sentado.

Se deberá informar si el esfuerzo realizado pareció ser significativo, considerando principalmente la frecuencia cardíaca y los cambios en escala de disnea y de fatiga de piernas.

Los valores medidos en pacientes y en sujetos sanos serán interpretados de acuerdo a las ecuaciones de referencia publicadas por Troosters $^{25}$ o Enright ${ }^{26}$ (anexo 6). La mayoría de los laboratorios en Chile actualmente utilizan los valores normales de Enright que podrán ser reemplazados por valores nacionales cuando éstos estén disponibles (observaciones no publicadas).

El informe deberá finalizar con una conclusión médica que integre globalmente los antecedentes clínicos con los valores obtenidos durante el examen. Se considerará normal un valor de distancia caminada mayor que el límite inferior (percentil 5) según la ecuación de referencia utilizada.
Si se cuenta con resultados de exámenes previos, se deberá considerar significativo un cambio en la distancia caminada mayor de 35 metros.

\section{Agradecimientos}

Al Sr. Vicente Macián, enfermero supervisor del laboratorio de función pulmonar del Hospital Universitario La Fe de Valencia y miembro del Comité Científico de la SEPAR, por la revisión crítica del manuscrito y por el aporte realizado a este instructivo durante el $41^{\circ}$ Congreso Chileno de Enfermedades Respiratorias.

\section{Bibliografía}

1.- COOPER K H. A means of assessing maximal oxygen intake: correlation between field and treadmill testing. JAMA 1968; 203: 201-4.

2.- McGAVIN C R, GUPTA S P, McHARDY G J. Twelveminute walking test for assessing disability in chronic bronchitis. BMJ 1976; 1: 822-3.

3.- BUTLAND R J A, PANG J, GROSS E R, WOODCOCK A A, GEDDES D M. Two-, six-, and 12-minute walking test in respiratory disease. BMJ 1982; 284: 1607-8.

4.- BITTNER V, WEINER D H, YUSUF S, ROGERS W J, MCINTYRE K M, BANGDIWALA S I, et al. Prediction of mortality and morbidity with a 6-minute walk test in patients with left ventricular dysfunction. JAMA 1993; 270: 1702-7.

5.- POULAIN M, DURAND F, PALOMBA B, CEUGNIET F, DESPLAN J, VARRAY A, et al. 6- Minute Walk Testing is more sensitive than Maximal Incremental Cycle Testing for detecting oxygen desaturation in patients with COPD. Chest 2003; 123: 1401-7.

6.- NIEDERMAN M S, CLEMENTE P H, FEIN A M, FEINSILVER S H, ROBINSON D A, ILOWITE J S, et al. Benefits of a multidisciplinary pulmonary rehabilitation program: improvements are independent of lung function. Chest 1991; 99: 798-804.

7.- KADIKAR A, MAURER J, KESTEN S. The six-minute walk test: a guide to assessment for lung transplantation. J Heart Lung Transplant 1997; 16: 313-9.

8.- CAHALIN L, PAPPAGIANOPOULOS P, PREVOST S, WAIN J, GINNS L. The relationship of the 6-min walk test to maximal oxygen consumption in transplant candidates with end-stage lung disease. Chest 1995; 108: 452-9.

9.- ATS Statement. Guidelines for the Six-Minute Walk Test. Am J Respir Crit Care Med 2002; 166: 111-7.

10.- PALANGE P, WARD S A, CARLSEN K-H, CASABURI R, GALLAGHER C G, GOSSELINK R, et al. ERS Task Force. Recommendations on the use of exercise testing in clinical practice. Eur Respir J 2007; 29: 185-209.

11.- BORG G A. Psycho-physical bases of perceived exertion. Med Sci Sports Exerc 1982; 14: 377-81.

12.- TROOSTERS T, VILARO J, RABINOVICH R, CASAS A, BARBERA J A, RODRÍGUEZ-ROISIN R, et al. Physiological responses to the 6-min walk test in patients with chronic obstructive pulmonary disease. Eur Respir J 2002; 20: 564-69. 
13.- COTE C G, CASANOVA C, MARÍN J M, LÓPEZ MV, PINTO-PLATA V, DE OCA M M, et al. Validation and comparison of reference equations for the 6-min walk distance test. Eur Respir J 2008; 31: 571-8.

14.- SZEKELY L A, OELBERG D A, WRIGHT C, JOHNSON D C, WAIN J, TROTMAN-DICKENSON $\mathrm{B}$, et al. Preoperative predictors of operative morbidity and mortality in COPD patients undergoing bilateral lung volume reduction surgery. Chest 1997; 111: 5508.

15.- PUHAN M A, MADOR M J, HELD U, GOLDSTEIN R, GUYATT G H, SCHÜNEMANN H J. Interpretation of treatment changes in 6-minute walk distance in patients with COPD. Eur Respir J 2008; 32: 637-43.

16.- CASANOVA C, COTE C G, MARÍN J M, DE TORRES J P, AGUIRRE-JAIME A, MÉNDEZ R, et al. The 6-min walking distance: long term follow up in patients with COPD. Eur Respir J 2007; 29: 535-40.

17.- CELLI B R, COTE C G, MARÍN J M, CASANOVA C, M DE OCA M, MÉNDEZ R A, et al. The body-mass index, airflow obstruction, dyspnea and exercise capacity index in chronic obstructive pulmonary disease. N Engl J Med 2004; 350: 1005-12.

18.- LISBOA C, BARRÍA P, YÁÑEZ J, AGUIRRE M, DÍAZ O. La prueba de caminata en seis minutos en la evaluación de la capacidad de ejercicio en pacientes con enfermedad pulmonar obstructiva crónica. Rev Méd Chile 2008; 136: 1056-64.

19.- MIYAMOTO S, NAGAYA N, SATOH T, KYOTANI S, SAKAMAKI F, FUJITA M, et al. Clinical correlates and prognostic significance of six-minute walk test in patients with primary pulmonary hypertension.
Comparison with cardiopulmonary exercise testing. Am J Respir Crit Care Med 2000; 161: 487-92.

20.- LEDERER D J, ARCASOY S M, WILT J S, D'OVIDIO F, SONETT J, KAWUT S M. Six-minute-walk distance predicts waiting list survival in idiopathic pulmonary fibrosis. Am J Respir Crit Care Med 2006; 174: 65964.

21.- McGOVERN J P, SASSE S A, STANSBURY D W, CAUSING L A, LIGHT R W. Comparison of oxygen saturation by pulse oximetry and co-oximetry during exercise testing in patients with COPD. Chest 1996; 109: 1151-5.

22.- CASANOVA C, COTE C, MARÍN J M, PINTO-PLATA V, DE TORRES J P, AGUIRRE-JAIME A, et al. Distance and oxygen desaturation during the 6-min walk test as predictors of long term mortality in patients with COPD. Chest 2008; 134: 746-52.

23.- LAMA V N, FLAHERTY K R, TOEWS G B, COLBY T V, TRAVIS W D, LONG Q, et al. Prognostic value of desaturation during a 6-minute walk test in idiopathic interstitial pneumonia. Am J Respir Crit Care Med 2003; 168: 1084-90.

24.- EATON T, YOUNG P, MILNE D, WELLS A U. Sixminute walk maximal exercise tests. Reproducibility in fibrotic interstitial pneumonia. Am J Respir Crit Care Med 2005; 171: 1150-7.

25.- TROOSTERS T, GOSSELINK R, DECRAMER M. Six minute walking distance in healthy elderly subjects. Eur Respir J 1999; 14: 270-4.

26.- ENRIGHT P L, SHERRILL D L. Reference equations for the six-minute walk in healthy adults. Am J Respir Crit Care Med 1998; 158: 1384-7.
Correspondencia a:

Mónica Gutiérrez Clavería

Hospital Naval, Viña del Mar

E-mail: mgancaroe@gmail.com 


\section{Anexo 1. Indicaciones escritas para los pacientes test de caminata en 6 min}

De preferencia venga acompañado

No haga ejercicio durante $\mathbf{2}$ horas antes de su examen

Mantenga sus medicamentos habituales

Coma y tome algo liviano en su horario habitual

Venga con ropa liviana y zapatos cómodos para caminar

\section{Anexo 2. Cuestionario para el paciente}

Nombre:

¿Usa Ud. medicamentos para enfermedades del corazón o respiratorias?

$\mathrm{Si}$

No

Anote el nombre del medicamento y hora en que los recibió hoy

Nombre

hora

¿Ha tenido Ud. dolor al pecho en los últimos 2 meses?

Sí

No

¿Ha tenido Ud. infarto al corazón en los últimos 2 meses?

Sí

No

Anexo 3. Escala de disnea de Borg

$\begin{array}{ll}0 & \text { NADA } \\ 0,5 & \text { MUY, MUY LEVE } \\ 1 & \text { MUY LEVE } \\ 2 & \text { LEVE } \\ 3 & \text { MODERADO } \\ 4 & \text { ALGO INTENSO } \\ 5 & \text { INTENSO } \\ 6 & \\ 7 & \text { MUY INTENSO } \\ 8 & \\ 9 & \\ 10 & \text { MUY, MUY INTENSO }\end{array}$




\section{Anexo 4. Estandarización del estímulo durante el examen}

El estímulo aumenta significativamente la distancia recorrida. Para lograr buena reproducibilidad del examen este estímulo debe estar estandarizado y debe ser realizado siempre igual.

1. Al iniciar el examen se debe decir al paciente que lo está haciendo "muy bien".

2. Al completar 1 minuto se le debe decir: "lo está haciendo bien, le quedan 5 minutos".

3. Al completar 2 minutos se le debe decir: "siga haciéndolo bien, le quedan 4 minutos".

4. Al completar 3 minutos se le debe decir: "lo está haciendo bien, ha completado la mitad del tiempo".

5. Al completar 4 minutos se le debe decir: "siga haciéndolo bien, le quedan sólo 2 minutos".

6. Al completar 5 minutos se le debe decir: "lo está haciendo bien, le queda sólo 1 minuto".

7. Si el paciente se detiene durante el examen y necesita descansar, se le debe decir: "puede apoyarse contra la pared si lo desea; continúe caminando en cuanto se sienta capaz de hacerlo".

8. Cuando falten 15 segundos se le debe decir: "en un momento le voy a indicar que se detenga donde esté, yo iré hasta donde usted se detuvo".

9. Al finalizar el examen se debe registrar al igual que al inicio la magnitud de la disnea y de fatiga de extremidades inferiores según la escala de Borg, cuidando de no influenciar el resultado.

10. Al finalizar la prueba es importante felicitar al paciente por su esfuerzo. No debe quedar con una mala experiencia después del examen.

11. Mientras el paciente descansa sentado, mídale la frecuencia cardíaca, la frecuencia respiratoria, la $\mathrm{SpO}_{2}$, la presión arterial, a los 2 y a los 5 min de terminada la caminata.

\section{Anexo 5 . Informe}

PRUEBA DE CAMINATA DE 6 MINUTOS

Nombre:

RUT:

Diagnóstico:

Fecha:

Edad: años

Estatura:

$\mathrm{cm}$

Peso: $\mathrm{kg}$

Presión sanguínea: ___ _ $/$ ___ _

Medicamentos tomados antes del examen:

Oxígeno suplementario durante el examen: NO:

SI: $\mathrm{L} / \mathrm{min}$.

\begin{tabular}{|l|l|l|l|}
\hline & Basal & Final & Recuperación 5 min \\
\hline Tiempo (hora, min.) & & & \\
\hline Frecuencia cardíaca (ciclos/min) & & & \\
\hline Frecuencia Respiratoria (ciclos/min) & & & \\
\hline Saturometría $\mathrm{O}_{2}(\%)$ & & & \\
\hline Disnea (Escala de Borg) & & & \\
\hline Fatiga (Escala de Borg) & & & \\
\hline
\end{tabular}

¿Se detuvo antes de los 6 minutos?

NO:

SI:

Razón:

Otros síntomas al finalizar el examen:

METROS CAMINADOS EN 6 min:

Conclusión:

*Referencia de valor teórico utilizado. **LIN= Límite inferior de normalidad. 


\section{Anexo 6. Valores de referencia para distancia caminada en 6 min}

- $\quad$ Ecuación de regresión de Troosters ${ }^{25}$

Hombre: $218+\left(5,14 \times\right.$ talla $_{\mathrm{cm}}-5,32 \mathrm{x}$ edad* $)-\left(1,8 \times\right.$ peso $\left._{\mathrm{kg}}+51,31\right)$

Mujer: $218+\left(5,14 \times\right.$ talla $_{\mathrm{cm}}-5,32 \times$ edad $\left.^{*}\right)-\left(1,8 \times\right.$ peso $\left._{\mathrm{kg}}\right)$

- $\quad$ Ecuación de regresión de Enright ${ }^{26}$

Hombre : $\left(7,57 \times\right.$ talla $\left._{\mathrm{cm}}\right)-(5,02 \times$ edad* $)-\left(1,76 \times\right.$ peso $\left._{\mathrm{kg}}\right)-309 \mathrm{~m}$

$\mathrm{LIN}=($ valor de referencia $-153 \mathrm{~m})$

Mujer : $\left(2,11 \times\right.$ talla $\left._{\mathrm{cm}}\right)-\left(5,78 \times\right.$ edad $\left.^{*}\right)-\left(2,29 \mathrm{x}^{\text {peso }} \mathrm{kg}_{\mathrm{g}}\right)+667 \mathrm{~m}$

$\mathrm{LIN}=($ valor de referencia $-139 \mathrm{~m})$

*Edad: en años

Ejemplos de valores de referencia según ecuación de Enright:

- $\quad$ Hombre de 50 años, talla $170 \mathrm{~cm}$, peso $70 \mathrm{~kg}$

V ref: 604 m (LIN $451 \mathrm{~m})$

- $\quad$ Mujer de 50 años, talla $160 \mathrm{~cm}$, peso $60 \mathrm{~kg}$

V ref: 578 m (LIN 439 m)

Vref: valor de referencia; LIN: límite inferior de normalidad 